\title{
A 4 × 4 Beamforming Network for Spatial Diversity and Multiplexing Reconfiguration
}

\author{
Li Sun (D), $^{1}$ Yuan Gao $\left(D^{2},{ }^{2}\right.$ Shi-gang Zhou, ${ }^{1}$ Guan-xi Zhang, ${ }^{3}$ and Gao-ang Li $^{4}$ \\ ${ }^{1}$ School of Microelectronics, Northwestern Polytechnical University, Xi'an 710072, China \\ ${ }^{2}$ School of Computer Science, Northwestern Polytechnical University, Xi'an 710072, China \\ ${ }^{3}$ Huawei Technologies Co., Ltd., Shanghai, China \\ ${ }^{4}$ Aerospace Information Research Institute, Chinese Academy of Sciences, Beijing, China \\ Correspondence should be addressed to Li Sun; lisun@nwpu.edu.cn and Yuan Gao; 2438399492@qq.com
}

Received 9 September 2021; Accepted 30 November 2021; Published 10 February 2022

Academic Editor: Mourad Nedil

Copyright ( $92022 \mathrm{Li}$ Sun et al. This is an open access article distributed under the Creative Commons Attribution License, which permits unrestricted use, distribution, and reproduction in any medium, provided the original work is properly cited.

\begin{abstract}
A simple $4 \times 4$ beamforming network with spatial diversity and multiplexing reconfiguration is proposed in this paper. By analyzing and decomposing the simplified transmission matrix of the network, it is found that the $4 \times 4$ butler matrix can achieve spatial diversity and multiplexing reconfiguration by switching the operating modes of the four-port devices. Therefore, by designing a reconfigurable device with three modes among hybrid coupler, crossover, and transmission line, a simple $4 \times 4$ reconfigurable beamforming network is achieved. The proposed network can achieve orthogonal spatial diversity responses with equal amplitude and progressive phase distribution responses at the four output ports (State 1) and hybrid spatial diversity and multiplexing responses (State 2) with equal amplitude and progressive phase distribution responses at the two adjacent output ports. For the experimental validation, a particular version of the reconfigurable device and network which work at $4.9 \mathrm{GHz}$, have been designed, fabricated, and tested. Good results indicate the proposed network can be widely used in wireless communication networks for MIMO applications.
\end{abstract}

\section{Introduction}

Nowadays, with the rapid development of wireless communication, the multiple-input multiple-output (MIMO) technique is adopted as a representative multiplexing radiation pattern forming technique in modern communication systems $[1,2]$. Spatial diversity and multiplexing are two important applications in MIMO systems. On one hand, transmit or receive diversity is a means to combat channel fading, providing higher directional gains and higher spectrum efficiency $[3,4]$. On the other hand, spatial multiplexing can increase the data transmission rate by transmitting independent information streams in parallel through the spatial channels, which is particularly important in the high-SNR regime where the system is degree-offreedom limited $[5,6]$. Therefore, a MIMO system usually provides two types of gains: spatial diversity gain and spatial multiplexing gain. Also, higher spatial multiplexing gains come at the price of sacrificing diversity and vice versa.

Reconfigurable multibeam antenna arrays have been widely used [7]. Typical reconfigurable multibeam antenna arrays consist of switches, a multibeam network, and an antenna array. Therefore, reconfigurable beam-forming networks have aroused extensive concern by scholars [8-11]. In [8], a reconfigurable $1 \times 4 \mathrm{RF}$ power distribution network has been designed. By changing the state of the variable phase shifters, the magnitude and phase relations among signals at the output ports can be modified. For squint-free beam steering, a reconfigurable series antenna feedline based on reconfigurable negative group delay circuits has been proposed [9], which has a tuning range from $-15^{\circ}$ to $15^{\circ}$ within a relative bandwidth of around 5\%. In [10], by controlling the RF signal path through the developed microstrip feedline network using PIN diodes, a pattern- 
reconfigurable microstrip patch array antenna with switchable beams is presented. A novel beam scanning antenna fed by a reconfigurable beam-forming network has been presented in [11]. In addition, to obtain better wireless communication performance in more complex environments, how to make a trade-off between diversity and multiplexing becomes more and more important. To the authors' best knowledge, such a multibeam network design topology for spatial diversity and multiplexing reconfiguration has not been presented earlier in the literature.

In this paper, a simple network for spatial diversity and multiplexing reconfiguration is presented. By analyzing and decomposing the simplified transmission matrix of the $4 \times 4$ butler matrix, it is concluded that by switching the operating modes of some four-port devices, the network can achieve reconfigurable output amplitude and phase responses between spatial diversity and multiplexing. Hence, a reconfigurable four-port device has been designed. It can switch the operating modes among hybrid coupler, crossover, and transmission line and is divided into two devices with two operating modes switching. Finally, by introducing reconfigurable devices, the simple $4 \times 4$ beam-forming network can work as a traditional $4 \times 4$ butler matrix (State 1) and two independent $2 \times 2$ orthogonal subnetworks in parallel (State 2 ) responses. As a theory validation, the proposed reconfigurable network with its reconfigurable device has been designed at a working frequency of $4.9 \mathrm{GHz}$. The whole network has been manufactured on a printed circuit board structure. The $S$ parameter measurement results show that the amplitude and phase of the output ports satisfy the requirements of the spatial diversity and multiplexing reconfiguration, which is in agreement with the simulations.

The paper is organized as follows: Section 2 analyzes the transmission matrix of a simple $4 \times 4$ butler matrix to achieve spatial diversity and multiplexing reconfiguration. Section 3 presents the detailed design of the proposed network, including the design of the reconfigurable device. Section 4 shows the measurements of the fabricated prototype. Finally, a conclusion is summarized in Section 5.

\section{Transmission Matrix Analysis of Simple Network}

As we all know, the ABCD matrix is only convenient for two-port network cascade connection calculation. While the scatter matrix is applicable for any $N$-port network, is not suitable for cascade connection. Different from the scatter matrix, the transmission matrix ( $T$ matrix) is particularly suited for cascading multiport networks. In order to reduce the complexity of matrix computing, simplified $T$ matrices and the matrix decomposition technique which is introduced in [12] are used for analyzing and designing the network to achieve spatial diversity and multiplexing reconfiguration.
For a $4 \times 4$ network with spatial diversity characteristics, the Butler matrix is the preferred beam-forming network. The T matrix decomposition of the $4 \times 4$ Butler matrix is as follows:

$$
\left[T_{1}\right]=\frac{1}{2}\left[\begin{array}{cccc}
e^{j(3 \pi / 4)} & e^{j(\pi / 2)} & e^{j(\pi / 4)} & 1 \\
e^{j(\pi / 2)} & e^{-j(\pi / 4)} & 1 & e^{j(\pi / 4)} \\
e^{j(\pi / 4)} & 1 & e^{-j(\pi / 4)} & e^{j(\pi / 2)} \\
1 & e^{j(\pi / 4)} & e^{j(\pi / 2)} & e^{j(3 \pi / 4)}
\end{array}\right]=A_{1} \cdot A_{2} \cdot A_{3} \cdot A_{4},
$$

where matrix $A_{1}$ is represented as a crossover for two middle ports, and its Tmatrix is a $4 \times 4$ permutation matrix shown as follows:

$$
A_{1}=\left[\begin{array}{llll}
1 & 0 & 0 & 0 \\
0 & 0 & 1 & 0 \\
0 & 1 & 0 & 0 \\
0 & 0 & 0 & 1
\end{array}\right] .
$$

Matrix $A_{2}$ and $A_{4}$ are both represented as two $3 \mathrm{~dB} / 90^{\circ}$ hybrid couplers, with the $T$ matrix shown as follows:

$$
A_{2}=A_{4}=\frac{1}{\sqrt{2}}\left[\begin{array}{cccc}
e^{j(\pi / 2)} & 1 & 0 & 0 \\
1 & e^{j(\pi / 2)} & 0 & 0 \\
0 & 0 & e^{j(\pi / 2)} & 1 \\
0 & 0 & 1 & e^{j(\pi / 2)}
\end{array}\right] \text {. }
$$

Matrix $A_{3}$ is represented as two $45^{\circ}$ phase shifters on side ports and a crossover on two middle ports, with the Tmatrix shown as follows:

$$
A_{3}=\frac{1}{\sqrt{2}}\left[\begin{array}{cccc}
e^{-j \frac{\pi}{4}} & 0 & 0 & 0 \\
0 & 0 & \sqrt{2} & 0 \\
0 & \sqrt{2} & 0 & 0 \\
0 & 0 & 0 & e^{-j \frac{\pi}{4}}
\end{array}\right] \text {. }
$$

In order to increase the transmission rate of wireless communication, switching the operating mode of the $4 \times 4$ network from spatial diversity to spatial multiplexing is a good choice. According to this kind of thought, a simple $4 \times 4$ network which is composed of two independent hybrid couplers and works as a $2 \times 2$ subnetwork can meet the requirements of spatial diversity and multiplexing. The $T$ matrix of the simple $4 \times 4$ network matrix is as follows: 


$$
\left[T_{\text {MIMO }}\right]=\frac{1}{\sqrt{2}}\left[\begin{array}{cccc}
0 & 0 & e^{j(\pi / 4)} & 1 \\
0 & 0 & e^{-j(\pi / 4)} & e^{j(\pi / 2)} \\
e^{j(\pi / 2)} & e^{-j(\pi / 4)} & 0 & 0 \\
1 & e^{j(\pi / 4)} & 0 & 0
\end{array}\right] \text {. }
$$

The $T$ matrix indicates that the simple $4 \times 4$ network can provide four beams, both of which have the same radiation coverage for spatial multiplexing. Through matrix decomposition and calculation, it is found that the Tmatrix of such a network can be decomposed as follows:

$$
\left[T_{2}\right]=B_{1} \cdot A_{2} \cdot A_{3} \cdot B_{4},
$$

where matrices $A_{2}$ and $A_{3}$ are represented as two $3 \mathrm{~dB} 90^{\circ}$ directional couplers and two $45^{\circ}$ phase shifters, shown in equations (3) and (4), respectively. Matrix $B_{1}$ is represented as four transmission lines with its $T$ matrix equal to unity, which is shown as follows:

$$
B_{1}=\left[\begin{array}{llll}
1 & 0 & 0 & 0 \\
0 & 1 & 0 & 0 \\
0 & 0 & 1 & 0 \\
0 & 0 & 0 & 1
\end{array}\right] .
$$

Matrix $B_{4}$ is represented as two crossovers for two side ports with its $T$ matrix shown as follows:

$$
B_{4}=\left[\begin{array}{llll}
0 & 1 & 0 & 0 \\
1 & 0 & 0 & 0 \\
0 & 0 & 0 & 1 \\
0 & 0 & 1 & 0
\end{array}\right] .
$$

Through the abovementioned analysis, it is concluded that by using the matrix decomposition technique on the $T$ matrix of the multiport network, the different operating modes can be obtained. The schematic diagram of this simple network is shown in Figure 1. For diversity and multiplexing reconfiguration, switching the operating modes of devices from $A_{1}$ and $A_{4}$ to $B_{1}$ and $B_{4}$, respectively, are better solutions. Hence, a simple network with diversity and multiplexing reconfiguration is achieved. By using RF switches, the reconfigurable four-port devices have been designed. In the next section, the reconfigurable device and the proposed simple network have been designed and analyzed.

\section{Design and Analysis of the Proposed Network}

Theoretically, the proposed network can be used at any frequency. For the experimental validation of the abovementioned theory, a particular version of the reconfigurable device and network have been designed. The working frequency has been chosen at $4.9 \mathrm{GHz}$, which is the new frequency band of $5 \mathrm{G}$ wireless communication. The prototype of the network is implemented in microstrip technology. At first, the reconfigurable device with operating modes among

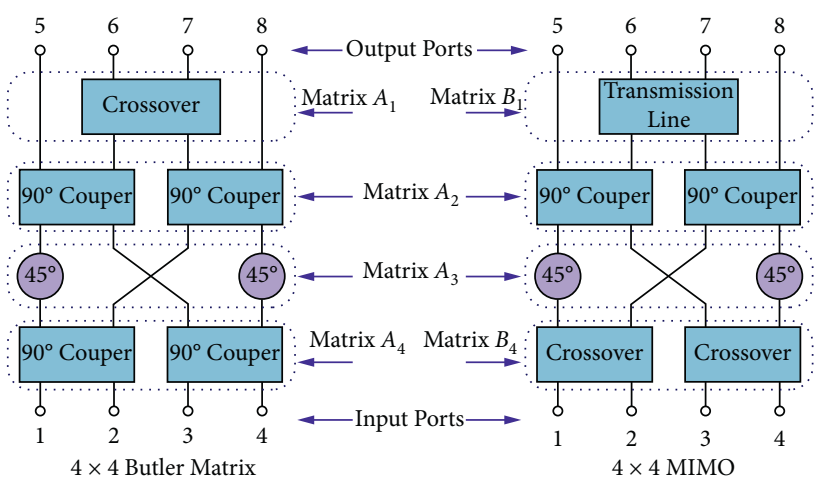

FIGURE 1: Schematic diagram of the proposed simple network.

$90^{\circ}$ hybrid coupler, crossover, and transmission line has been designed at a working frequency of $4.9 \mathrm{GHz}$. Then, the proposed reconfigurable network is implemented based on the reconfigurable device.

3.1. Design of the Reconfigurable Device. The schematic diagram of the reconfigurable device is shown in Figure 2. It is printed on a substrate with a dielectric constant of 3.66 and a thickness of $0.762 \mathrm{~mm}$. Its detailed parameters are shown in Table 1. It is designed based on a three-section branch-line crossover [13], possessing a simple symmetrical structure. In order to operate as a hybrid coupler, two fork-shaped strips are added symmetrically on the middle branch lines. The fork-shaped strips provide capacitive loading that can change the direction and distribution of currents, thereby obtaining a hybrid coupler of the device. On the other hand, when the middle branch lines are shorted to the ground, the proposed device can work as two independent transmission lines. Therefore, the proposed device can obtain reconfigurable working modes among $90^{\circ}$ hybrid coupler, crossover, and transmission line with the ideal RF switches connecting fork-shaped strips, turning them off and shorting them to the ground, corresponding to its T matrix switching among $A_{4}, B_{4}$, and $B_{1}$, respectively.

The simulated results of the reconfigurable device with three different working modes are shown in Figure 3. Figure 3(a) shows the $3 \mathrm{~dB} 90^{\circ}$ hybrid coupler mode with the RF switches connecting the fork-shaped strips. The proposed device exhibits return loss and port isolation of less than $-20 \mathrm{~dB}$, a power division ratio of $3.1 \pm 0.2 \mathrm{~dB}$, and an output phase difference of $90 \pm 1^{\circ}$ between 4.7-5.2 GHz. As shown in Figure 3(b), when the middle branch lines are connected to nothing, which means the RF switches are turned off, the proposed device works as a crossover with both return loss and isolation below $-20 \mathrm{~dB}$ from $4.75-5 \mathrm{GHz}$. The transmission coefficient of the cross port is about $-0.3 \mathrm{~dB}$. As shown in Figure 3(c), when the middle branch lines are shorted to the ground, the proposed device works as two independent transmission lines with both return loss and port isolation below $-22 \mathrm{~dB}$ from $4.7-5 \mathrm{GHz}$. While the insertion loss of the transmission port is about $-0.2 \mathrm{~dB}$, from the result, we can see that the device can switch the operating modes among the $3 \mathrm{~dB} 90^{\circ}$ hybrid coupler, crossover, and transmission line, satisfying the design requirements. 


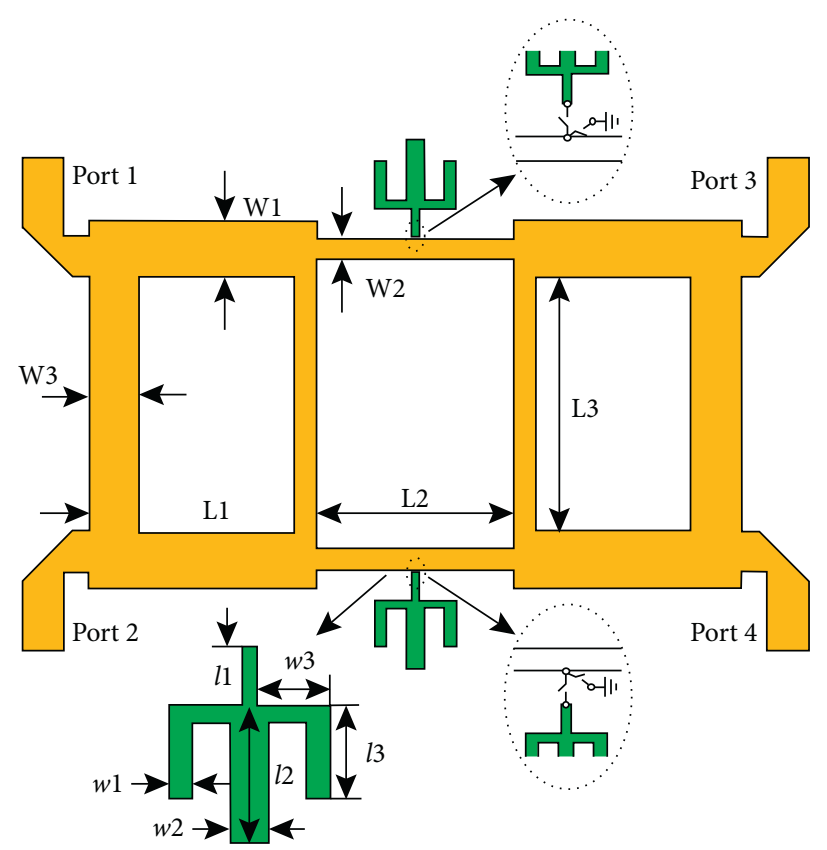

FIgURE 2: Schematic diagram of the reconfigurable device.

TABLE 1: Detailed parameters of the multifunctional component.

\begin{tabular}{lcccccc}
\hline Parameters & $W 1$ & $L 1$ & $W 2$ & $L 2$ & $W 3$ & $L 3$ \\
Values $(\mathrm{mm})$ & 2.3 & 6.4 & 0.9 & 8 & 2 & 10.4 \\
Parameters & $w 1$ & $l 1$ & $w 2$ & $l 2$ & $w 3$ & $l 3$ \\
Values (mil) & 0.4 & 1.2 & 0.8 & 2.5 & 1.5 & 1.9 \\
\hline
\end{tabular}

For achieving two reconfigurable devices based on Figure 1, the proposed reconfigurable device has been redesigned into two different reconfigurable devices based on whether there are fork-shaped strips. The schematic diagram of two reconfigurable devices is shown in Figure 4. With the RF switches turned on and off, Device A can change operating modes between the $3 \mathrm{~dB} 90^{\circ}$ hybrid coupler and crossover. On the other hand, Device B can change operating modes between the transmission line and crossover by turning on and off RF switches. Therefore, in practical fabrication, the RF switches can be achieved by PIN diodes. In the next part, the proposed whole network is designed based on the reconfigurable device.

3.2. Design of the Proposed Network. Based on the proposed reconfigurable device, a simple reconfigurable network has been designed. The schematic of the network is shown in Figure 5. It is printed on a substrate with a dielectric constant of 3.66 and a thickness of $0.762 \mathrm{~mm}$. The proposed network consists of reconfigurable devices (two Device A and one Device B), a crossover, two $90^{\circ}$ hybrid couplers, and transmission lines. The $90^{\circ}$ hybrid couplers are implemented with $3 \mathrm{~dB}$ branch lines. Eventually, when the red-marked RF switches turn on (Device A with $3 \mathrm{~dB} 90^{\circ}$ hybrid coupler mode) and the blue-marked RF switches turn off (Device B with crossover mode), the whole network works at State 1, obtaining spatial diversity with four beams in a different direction. On the other hand, when the red-marked RF switches turn off (Device A with crossover mode) and the blue-marked RF switches turn on (Device B with transmission mode), the proposed network works at State 2, obtaining multiplexing with four beams two of which have the same direction.

The simulated $S$-parameter results of the proposed network with both State 1 and State 2 are shown in Figures 6-8. Considering the symmetry of the network, some result curves are not shown. From the results we can see, the network exhibits reflection coefficients of all ports less than $-15 \mathrm{~dB}$ from $4.87-4.96 \mathrm{GHz}$ for both State 1 and State 2 . When the network works at State 1, the power of the input port is equally divided into four output ports, with a power division ratio of $6.5 \pm 0.5 \mathrm{~dB}$ and the port isolation of less than $-20 \mathrm{~dB}$ over the operating frequency band. When the network works at State 2, the power of the input port is equally divided into two corresponding output ports, with a power division ratio of $3.3 \pm 0.5 \mathrm{~dB}$ and a port isolation of less than $-20 \mathrm{~dB}$ over the operating frequency band. Figure 8 shows the simulated phase responses of the simple network. The detailed phase results are shown in Tables 2 and 3. When the network works at State 1, as shown in Figure 8(a), the adjacent two output ports have the same phase difference of $45^{\circ},-135^{\circ}, 135^{\circ}$, and $-45^{\circ}$ with Port 1 , Port 2 , Port 3 , and Port 4 excited, respectively. Therefore, four radiation beams with four orthogonal beam points have been obtained for spatial diversity application. On the other hand, when the network works at State 2, as shown in Figure 8(b), the corresponding two output ports have a phase difference of $90^{\circ}, 90^{\circ},-90^{\circ}$, and $-90^{\circ}$ with Port 1 , Port 2, Port 3, and Port 4 excited, respectively. Hence, four radiation beams with two same beam points have been obtained for spatial multiplexing application. 


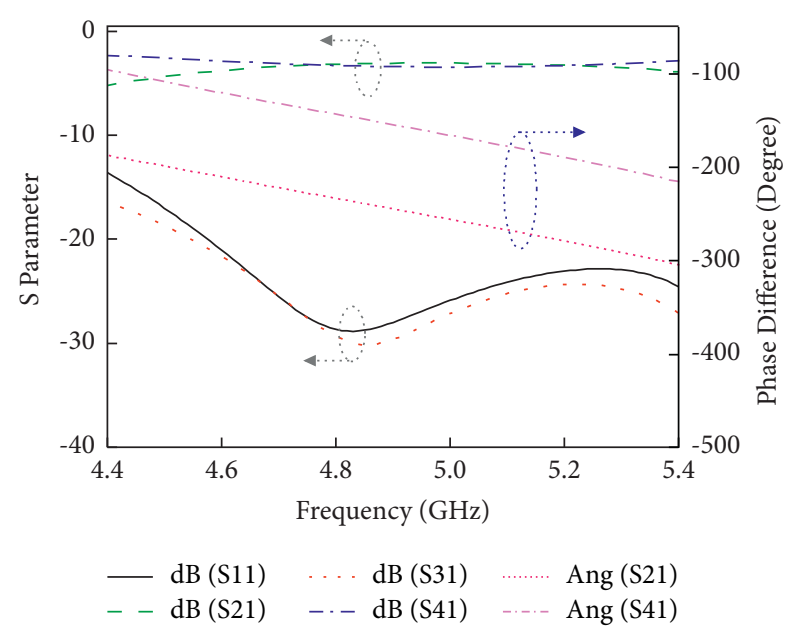

(a)

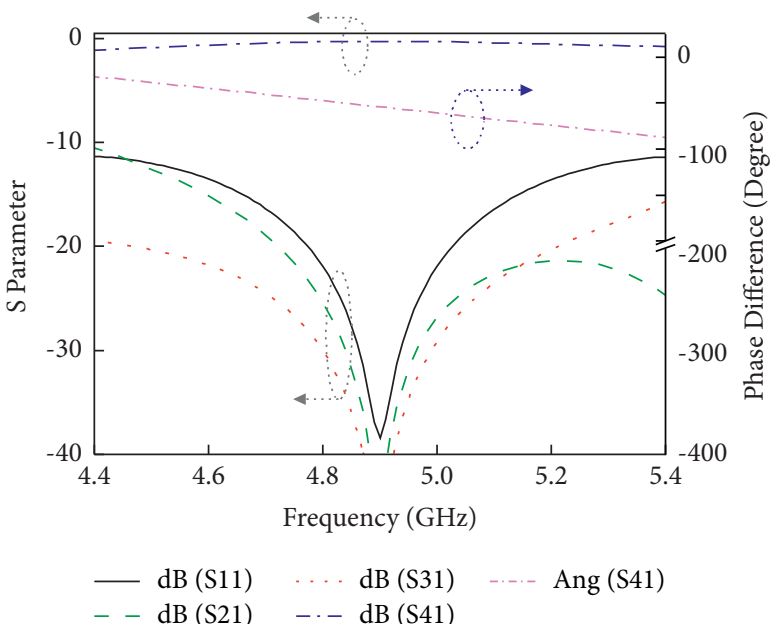

(b)

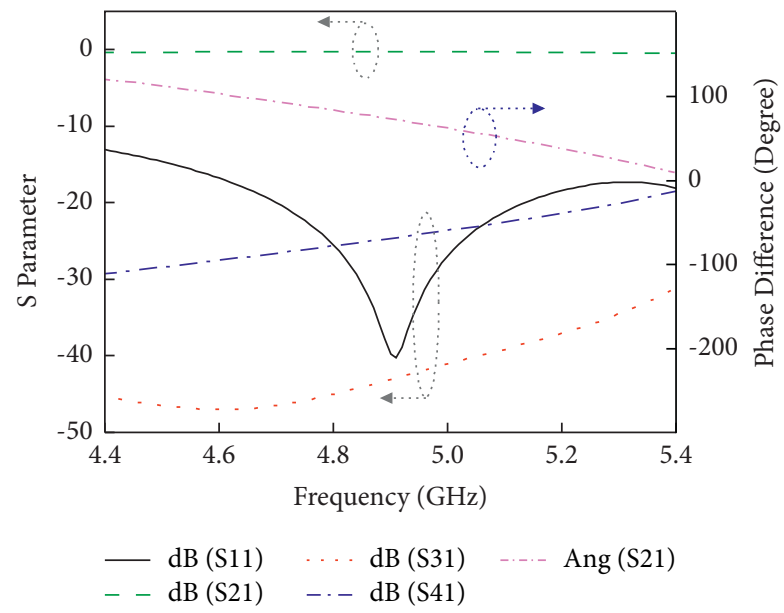

(c)

FIGURE 3: Simulated $S$ parameter results with different working states of the reconfigurable device: (a) $3 \mathrm{~dB} 90^{\circ}$ hybrid coupler with the RF switches connecting the fork-shaped strips; (b) crossover with the RF switches turned off; (c) transmission line with the RF switches connecting the ground.

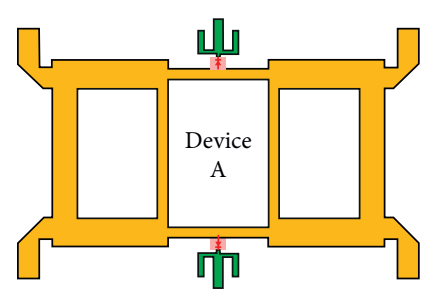

(a)

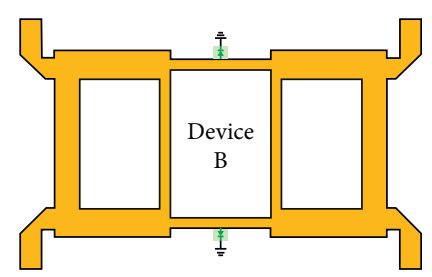

(b)

Figure 4: Schematic diagrams of two reconfigurable devices: (a) Device A and (b) Device B.

The abovementioned simulation results show that the proposed $4 \times 4$ beamforming network can achieve orthogonal spatial diversity responses with equal amplitude and progressive phase distribution responses at the four output ports (State 1) and hybrid spatial diversity and multiplexing responses (State 2) with equal amplitude and progressive phase distribution responses at the two adjacent output ports.

\section{Measured Results of the Proposed Network}

4.1. Fabrication and Measured Results of the Proposed Network. Based on the design of the proposed simple network in Section 3, the network has been fabricated and measured. The network is printed on a substrate of Rogers 4350 with a dielectric constant of 3.66 and a thickness of $0.762 \mathrm{~mm}$. As for RF switches, commercial PIN diodes are 


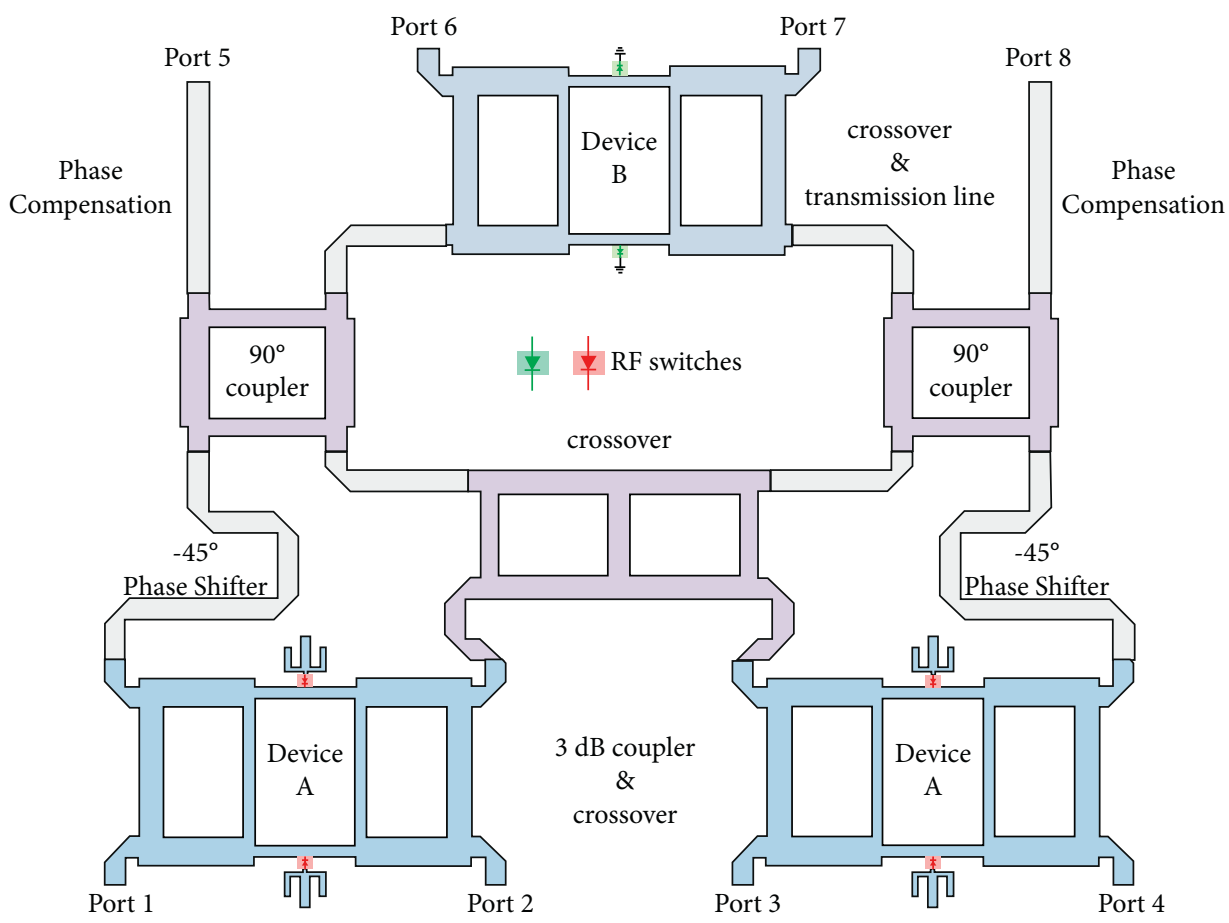

FIgURE 5: Schematic diagram of the proposed reconfigurable network. The red and blue RF diodes are the same type of RF diode. Color discrimination indicates different working states of the RF diodes.

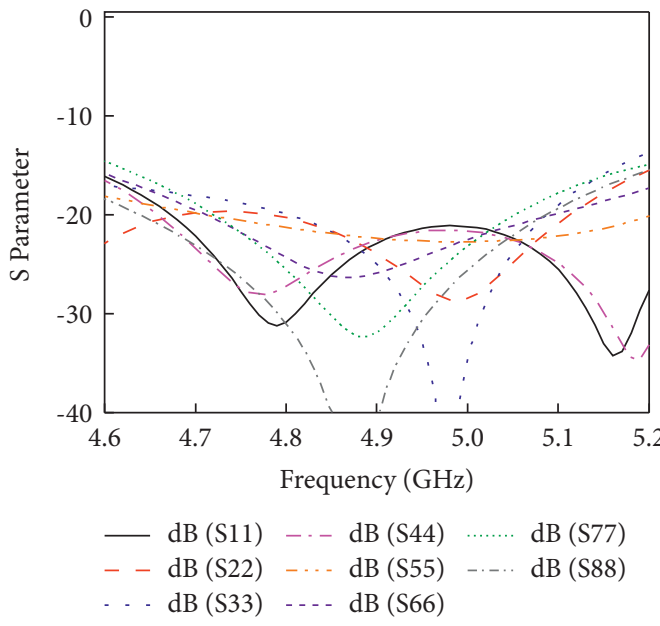

(a)

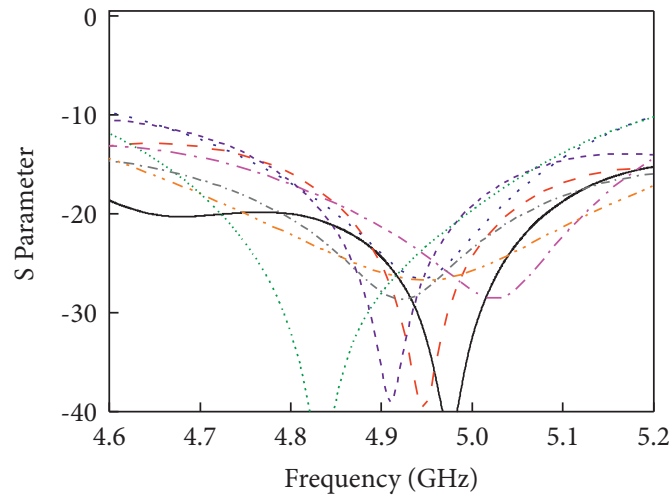

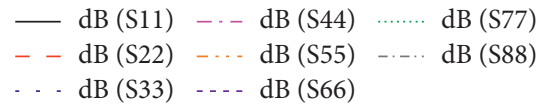

(b)

Figure 6: Simulated return losses when the simple network works at (a) State 1 and (b) State 2.

adopted to select the proper working mode of the reconfigurable devices, model MEST2G-010-20 from M/A-COM [14]. It shows high isolation of $-18 \mathrm{~dB}$ and low insertion loss of $0.35 \mathrm{~dB}$ around a working frequency of $4.9 \mathrm{GHz}$, which meets the requirements. The picture of the fabricated network is shown in Figure 9. As can be seen, 6 PIN diodes are soldered to the substrate.

The measured $S$-parameter results of the proposed network with both State 1 and State 2 are shown in Figures 10-12. The slight discrepancy between the simulation and measurement is due to fabrication errors and the effects of PIN diodes. From the results we can see, the network exhibits reflection coefficients of all ports less than $-15 \mathrm{~dB}$ from $4.9-4.96 \mathrm{GHz}$ for both State 1 and State 2. While its reflection coefficients are less than $-10 \mathrm{~dB}$, the frequency range for both State 1 and State 2 is 4.75-5.13 GHz. The measured port insert loss, isolation, and phase difference for State 1 are shown in Figure 11. Because of the symmetry of the network, parts of the results are not shown. When the network works at State 1, the power of the input port is equally divided into four output ports, with a power division ratio of $7 \pm 0.5 \mathrm{~dB}$ and a port isolation of less than $-16 \mathrm{~dB}$ over the operating frequency band. The increased insert loss is due to 


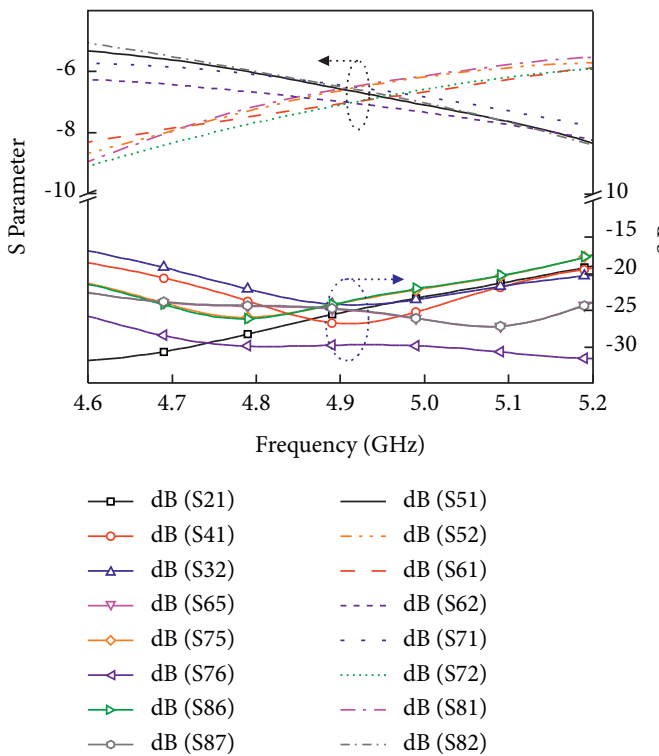

(a)

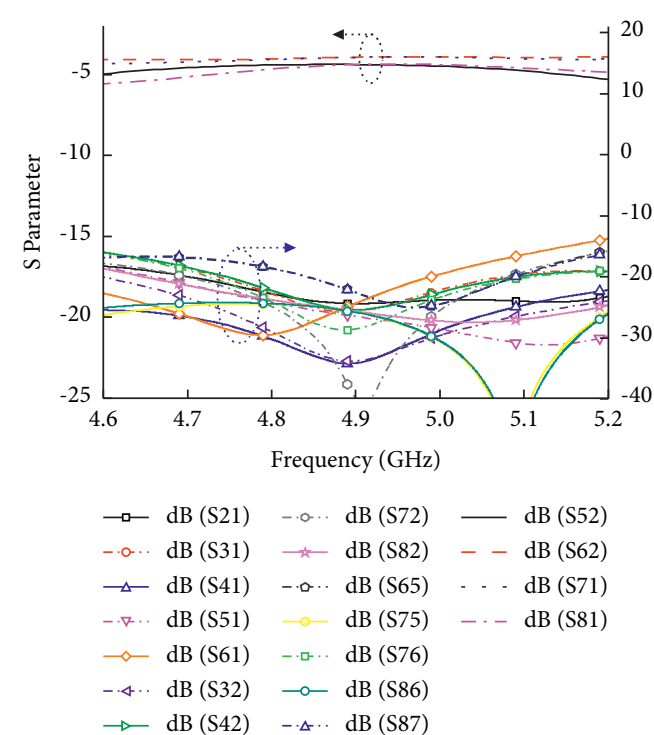

(b)

FiguRE 7: Simulated transmission parameters when the simple network works at (a) State 1 and (b) State 2.
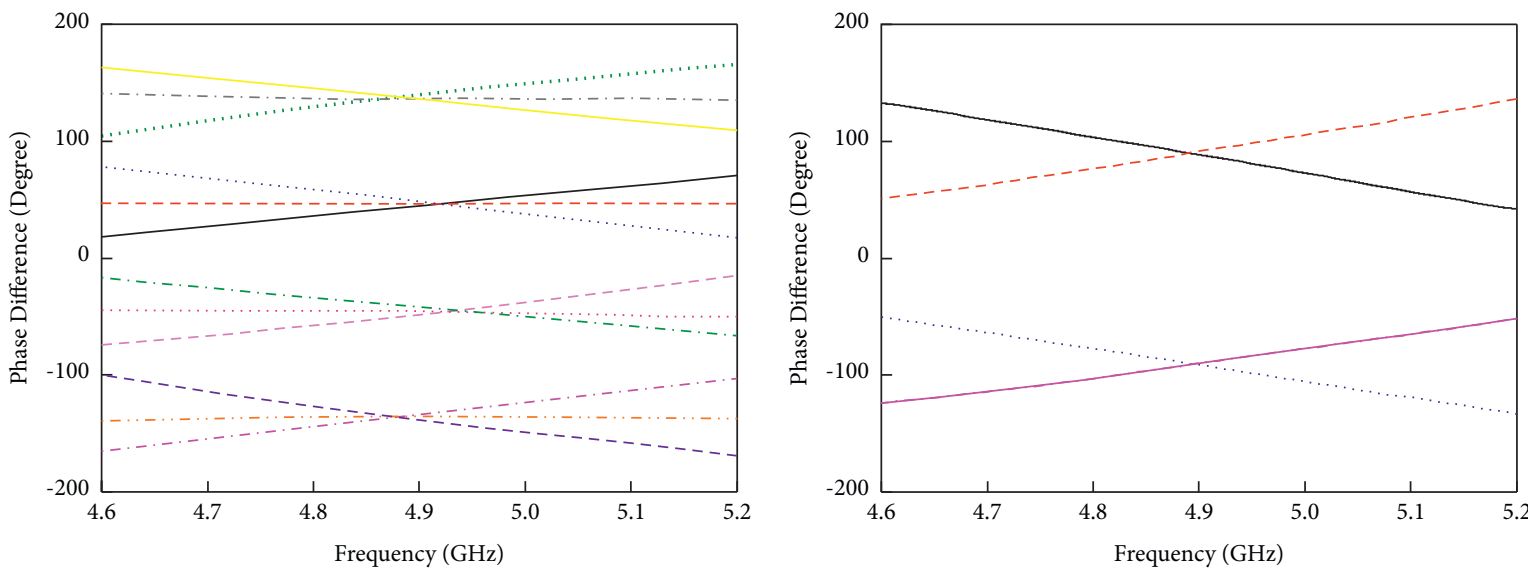

\begin{tabular}{|c|c|}
\hline An & A \\
\hline & $-{ }_{-A}$ \\
\hline & 883) \\
\hline ) & 64) \\
\hline ) & Ang (S64)-A \\
\hline Ang (S72)-Ang (S82) & Ang (S74)-Ang (S84) \\
\hline
\end{tabular}

(a)

(b)

FIgURE 8: Simulated phase response when the simple network works at (a) State 1 and (b) State 2.

TABLE 2: Phase relations of the inputs and outputs for the network working at State 1 (unit: degree).

\begin{tabular}{lccccc}
\hline Ports & Port 5 & Port 6 & Port 7 & Port 8 & $\Delta \varphi$ \\
\hline Port 1 & -217 & -259 & -305 & -350 & $45 \pm 3$ \\
Port 2 & -127 & 5 & -217 & -85 & $-135 \pm 5$ \\
Port 3 & -86 & 143 & 3.6 & -127 & $135 \pm 5$ \\
Port 4 & -353 & -310 & -263 & -220 & $-45 \pm 3$ \\
\hline
\end{tabular}

TABle 3: Phase relations of the inputs and outputs for the network working at State 2 (unit: degree).

\begin{tabular}{|c|c|c|c|c|c|}
\hline Ports & Port 5 & Port 6 & Port 7 & Port 8 & $\Delta \varphi$ \\
\hline Port 1 & - & - & 56 & -36 & $90 \pm 3$ \\
\hline Port 2 & -13 & -100 & - & - & $90 \pm 3$ \\
\hline Port 3 & - & - & -96 & -7 & $-90 \pm 3$ \\
\hline Port 4 & -53 & 37 & - & - & $-90 \pm 3$ \\
\hline
\end{tabular}




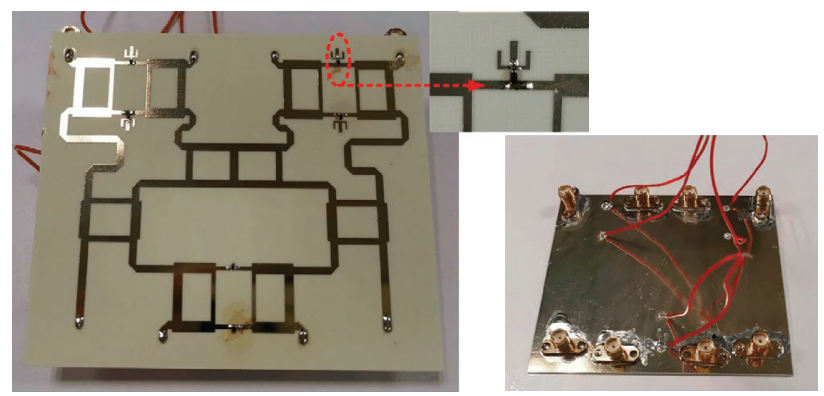

(a)

(b)

FIGURE 9: Fabrication of the proposed reconfigurable network.

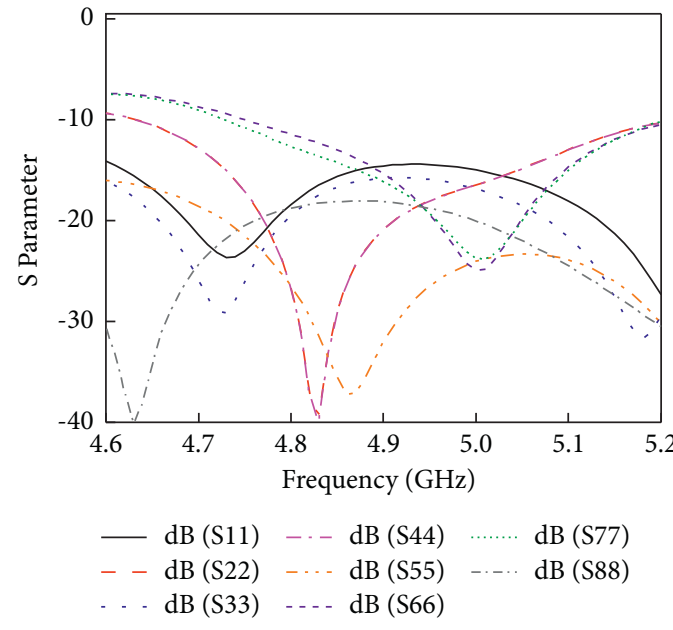

(a)

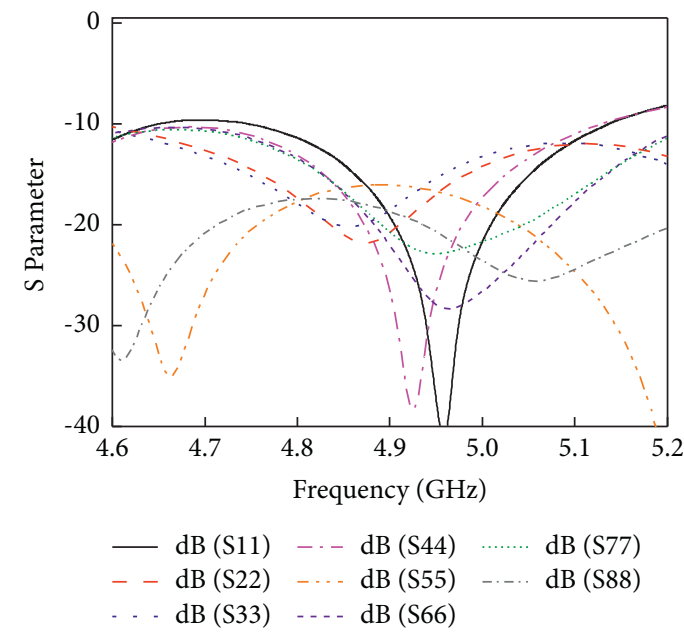

(b)

FIgURE 10: Measured return losses when the simple network works at (a) State 1 and (b) State 2.

the introduction of PIN diodes. The phase differences at the center frequency of $4.9 \mathrm{GHz}$ are $45^{\circ},-135^{\circ}, 135^{\circ}$, and $-45^{\circ}$ with Port 1, Port 2, Port 3, and Port 4 excited, respectively. The differential phase imbalance does not exceed $\pm 10^{\circ}$, which is in good agreement with the simulation ones. The measured port phase and amplitude for State 2 are shown in Figure 12. As can be seen, the power of the input port is equally divided into two corresponding output ports, with a power division ratio of $4.3 \pm 0.2 \mathrm{~dB}$, and the port isolations are less than $-18 \mathrm{~dB}$ over the operating frequency band. The phase differences at the center frequency of $4.9 \mathrm{GHz}$ are $96^{\circ}, 84^{\circ},-84^{\circ}$, and $-96^{\circ}$ with Port 1 , Port 2, Port 3, and Port 4 excited, respectively. The differential phase imbalance does not exceed $\pm 10^{\circ}$ over the whole working frequency band, which is in good agreement with the simulation ones.

4.2. Application on an Antenna Array. To validate the diversity and multiplexing reconfiguration performance of the novel network, the network should be connected to an antenna array to test the radiation patterns. Considering the antenna array performance, a printed Yagi antenna array was chosen as the radiation structure. The geometry of the $1 \times 4$ antenna array is shown in Figure 13, which is based on the printed Yagi antenna. The radiating elements have been spaced $32 \mathrm{~mm}$ apart from center to center, which corresponds to $0.53 \lambda$ at $5 \mathrm{GHz}$. In order to increase the isolation between the adjacent ports, bended microstrips are added between the elements. The $\mathrm{S}$ parameters of the proposed antenna array are shown in Figure 14. As can be seen, return losses and isolation are both less than $-19 \mathrm{~dB}$ over the working frequency range of 4.6-5.2 GHz.

The proposed Yagi antenna array is connected with the input ports of the novel network, which is simulated through the cosimulation of the electromagnetic field and circuit of ANSYS HFSS software. For testing the $S$-parameters of the beamforming antenna array, the proposed beamforming network is connected with the antenna array by coaxial lines of the same length, as shown in Figure 15. There is a little difference between the simulation and measured results due to the influence of PIN diodes. The return losses of the reconfigurable multibeam antenna array network are shown in Figure 16, which are less than $-12 \mathrm{~dB}$ over the working frequency from 4.9-5.15 GHz for the network at both State 1 and State 2. The isolation is shown in Figure 17. When the network works at State 1 and State 2, the ports' isolation is less than $-15 \mathrm{~dB}$ and $-18 \mathrm{~dB}$, respectively. The normalized radiation patterns in the E-plane at a working frequency of $5 \mathrm{GHz}$ for each port are shown in Figure 18. It can be observed when the network works at State 1, the four 


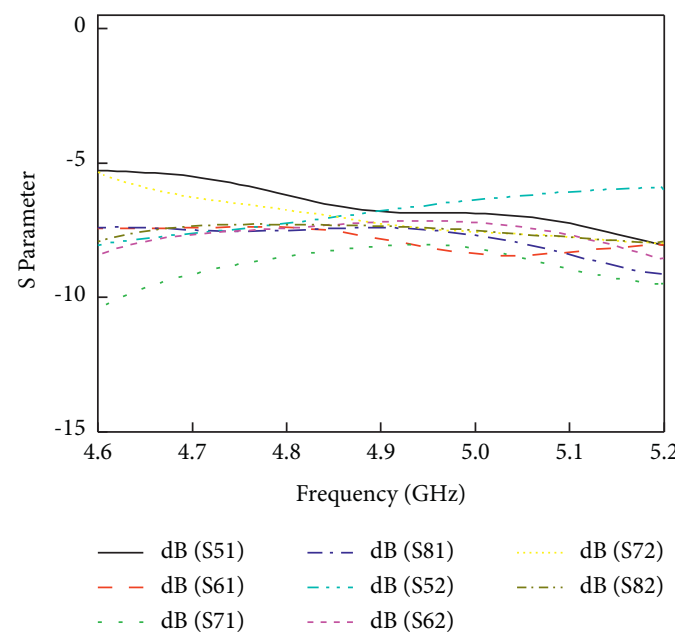

(a)

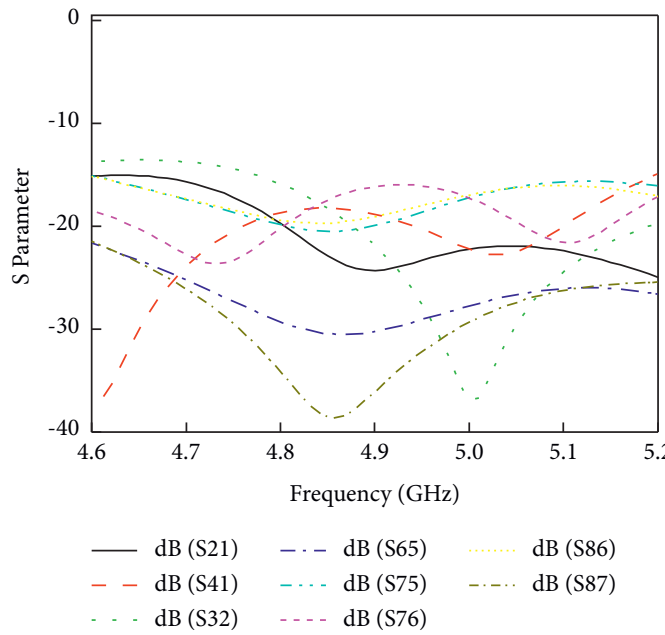

(b)

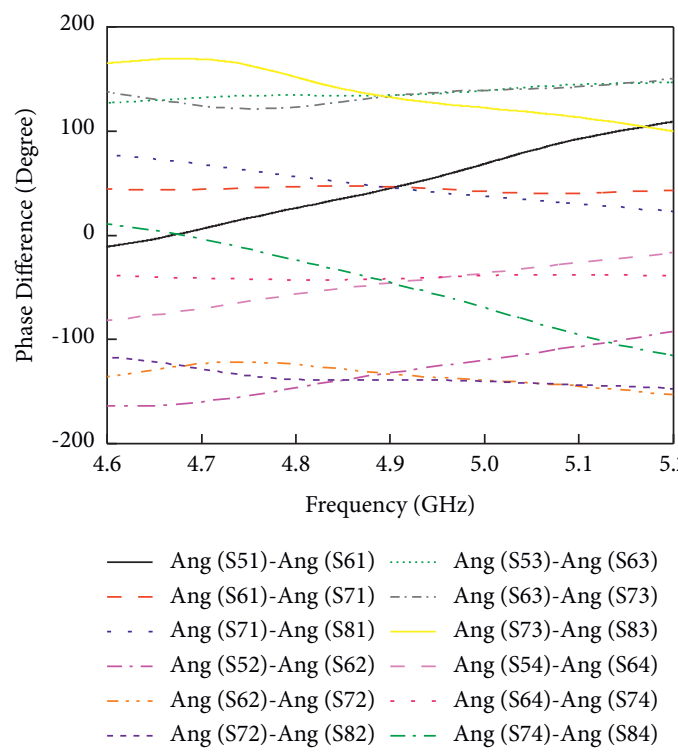

(c)

Figure 11: Measured (a) insert loss, (b) isolation, and (c) phase difference when the simple network works at State 1.

orthogonal beams are obtained, with directions of $145^{\circ}, 45^{\circ}$, $-45^{\circ}$, and $-145^{\circ}$ for Port 1 , Port 2, Port 3, and Port 4, respectively. When the network works at State 2 , the beam directs at $-45^{\circ}$ when Port 1 and Port 2 are excited, while the beam directs at $45^{\circ}$ when Port 3 and Port 4 are excited. The radiation patterns of the beamforming antenna array have not been measured because it is not convenient to place a DC signal generator in the microwave anechoic chamber. However, the actual radiation patterns should agree with the simulations due to the good $S$-parameters. All these results suggest that with the reconfigurable radiation patterns, the proposed reconfigurable multibeam antenna array network system can be well operated in dynamic environments.

Finally, a comparison of reconfigurable performance among this proposed beamforming network and several designs as abovementioned is tabulated in Table 4. It is found that only the proposed beamforming network can obtain both spatial diversity and multiplexing performance. 

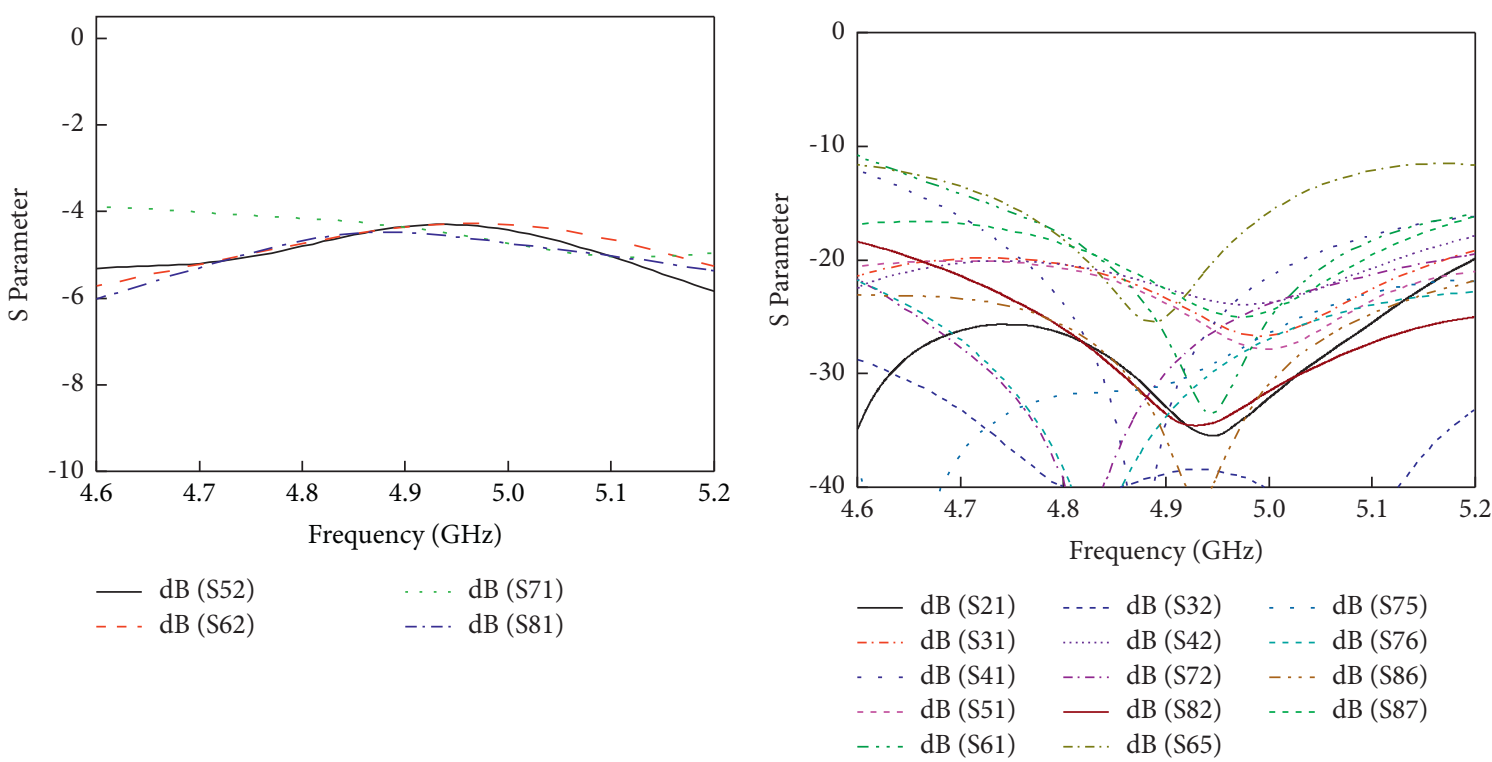

(a)

(b)

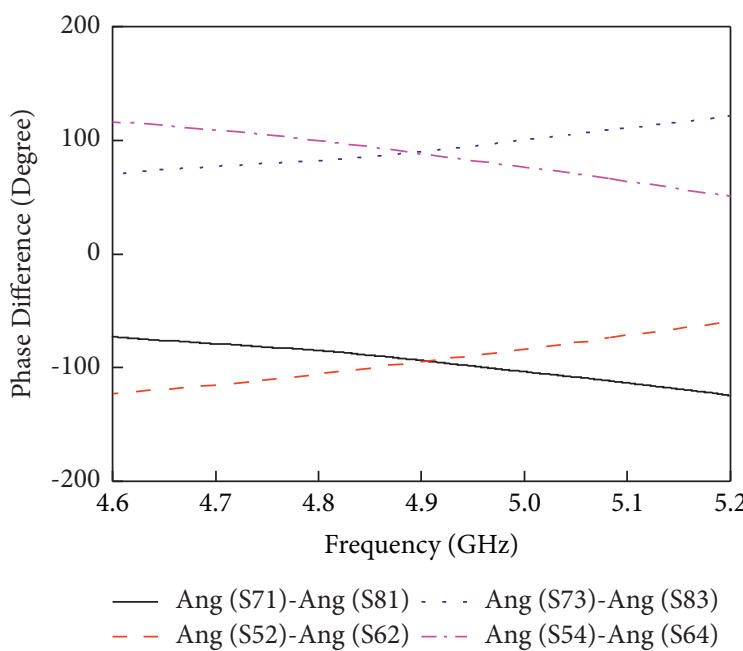

(c)

Figure 12: Measured (a) insert loss, (b) isolation, and (c) phase difference when the simple network works at State 2.

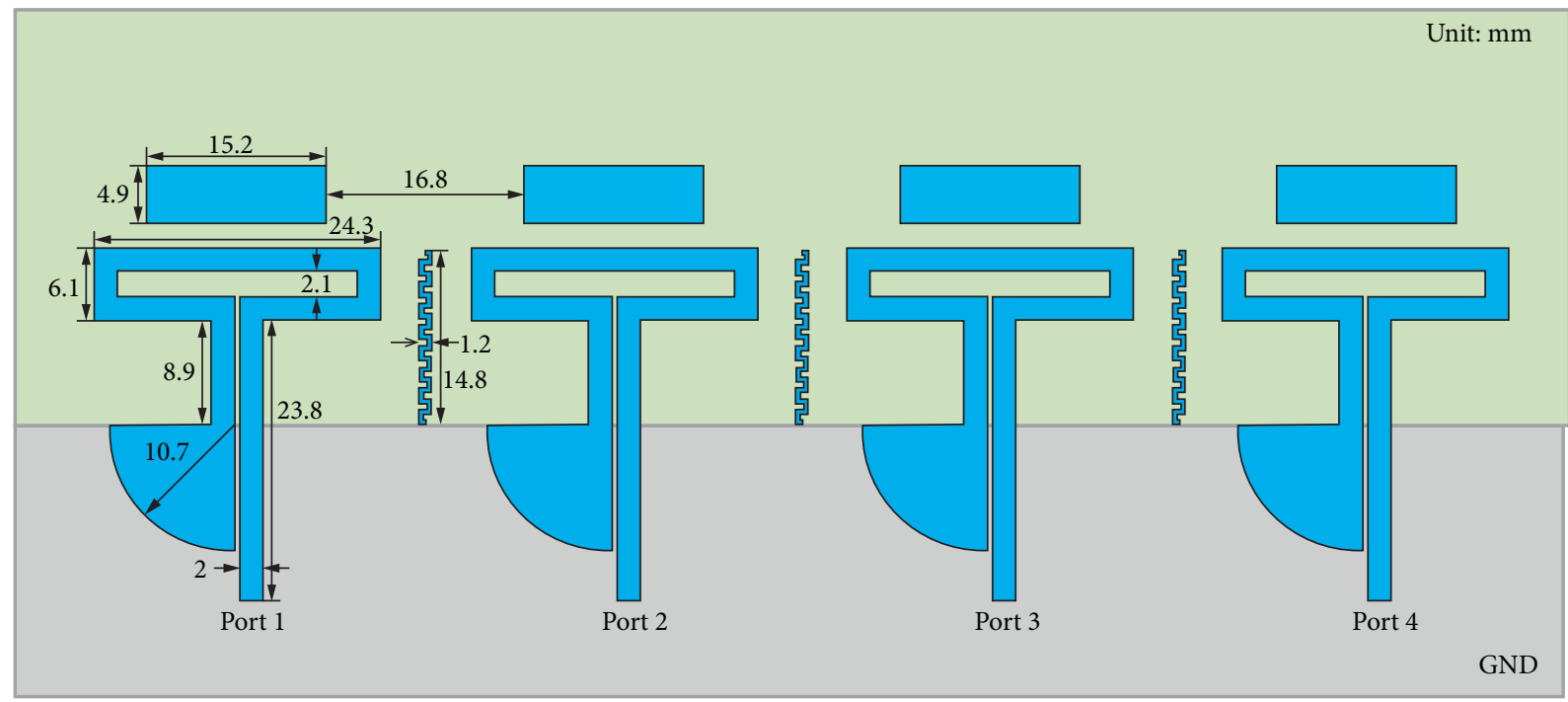

Figure 13: Geometry of the Yagi antenna array. 


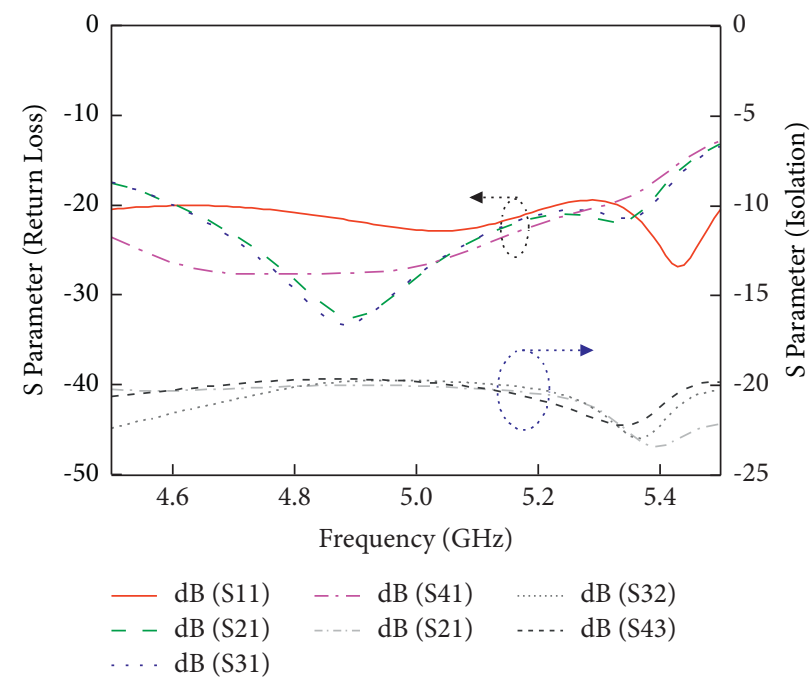

FIGURE 14: Simulated and measured $S$ parameter of the Yagi antenna array.

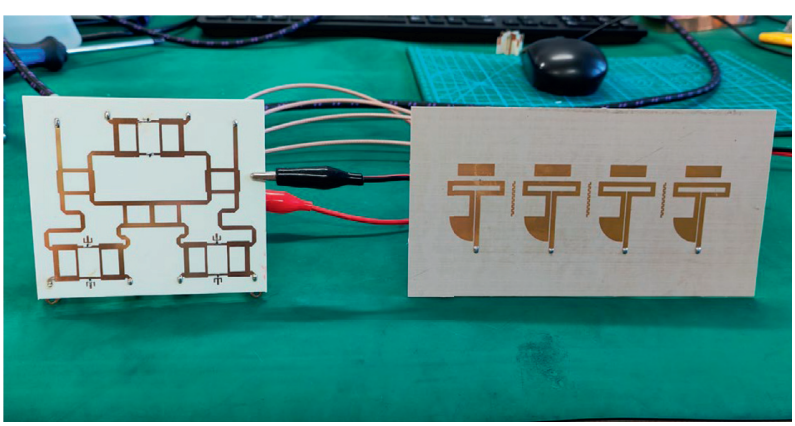

Figure 15: Photograph of the reconfigurable beamforming antenna array.

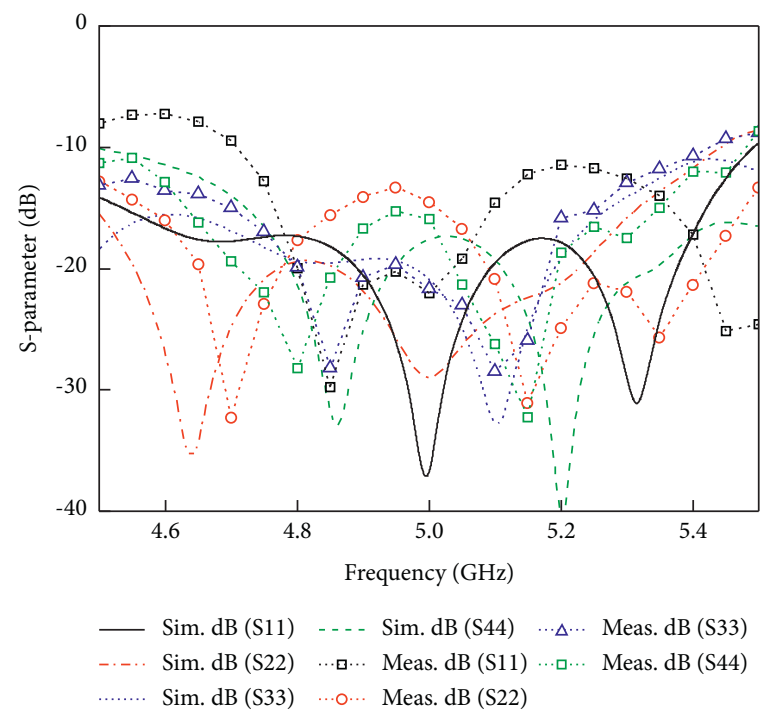

(a)

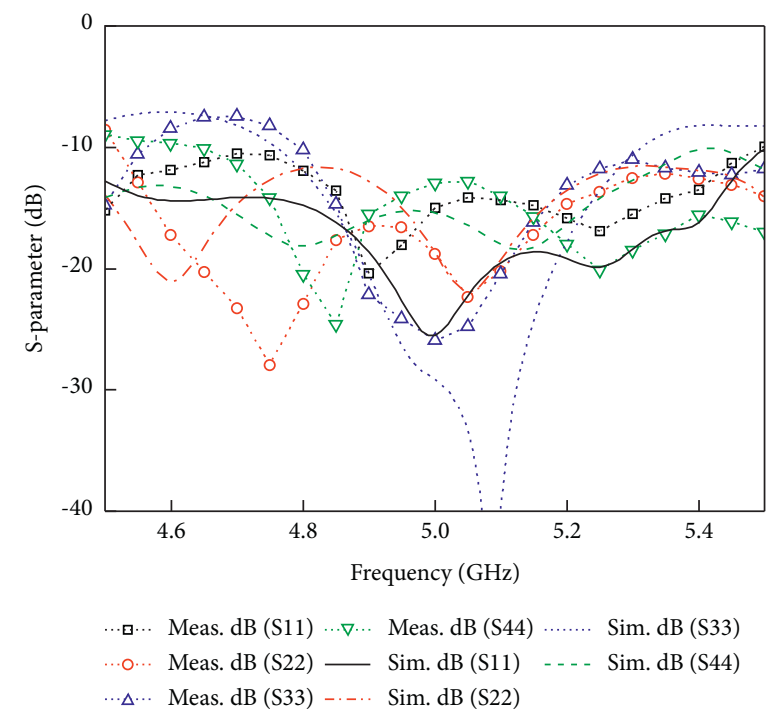

(b)

FIGURE 16: Simulated and measured return losses of the whole reconfigurable antenna array when the network is at (a) State 1 and (b) State 2. 


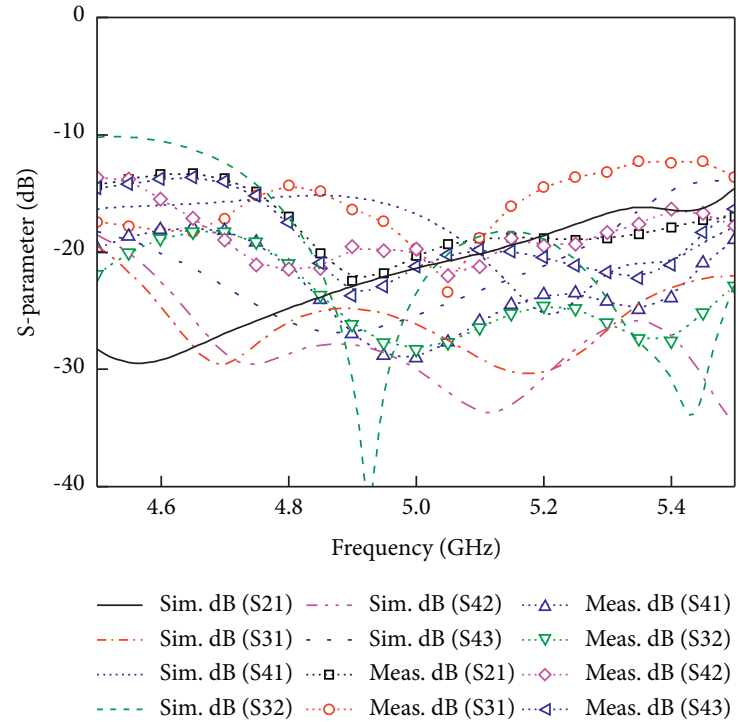

(a)

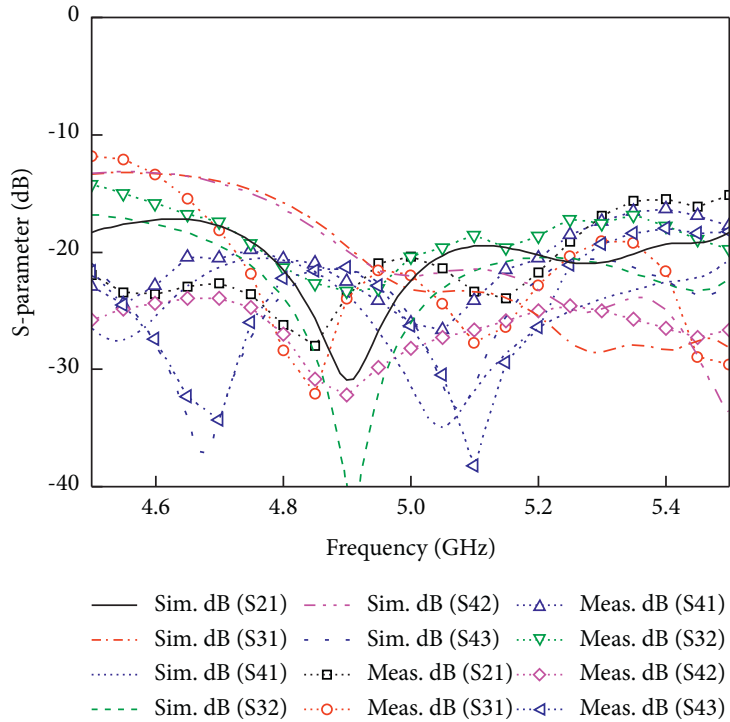

(b)

FIgURE 17: Simulated and measured isolation of the whole reconfigurable antenna array when the network is at (a) State 1 and (b) State 2.

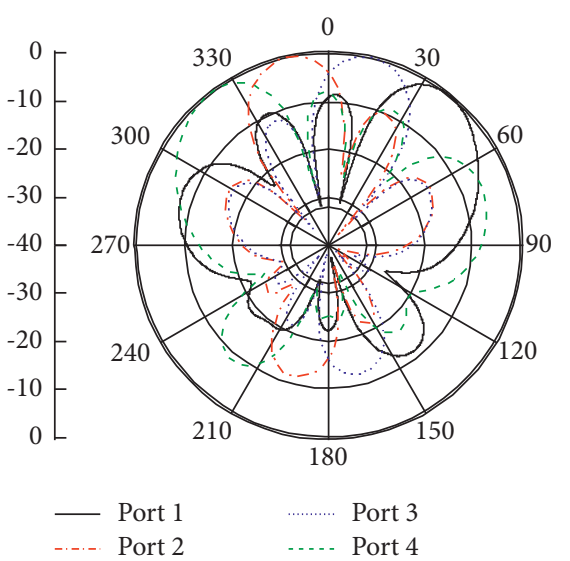

(a)

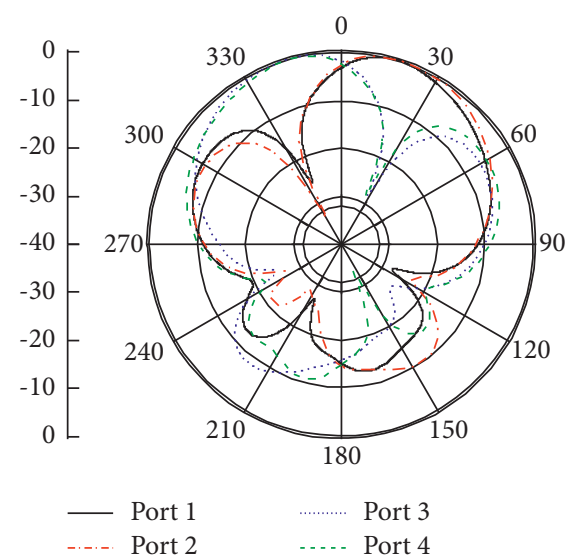

(b)

FiguRe 18: Simulated radiation patterns of the whole reconfigurable antenna array when the network is at (a) State 1 and (b) State 2.

TABLE 4: Comparison of the reconfigurable beamforming network.

\begin{tabular}{lcc}
\hline Ref. & Network port number & Reconfigurable performance \\
\hline$[8]$ & $1 \times 4$ & Spatial diversity \\
{$[9]$} & $1 \times 2$ & Spatial diversity \\
{$[10]$} & $1 \times 2$ & Spatial diversity \\
{$[11]$} & $1 \times 11$ & Spatial diversity \\
This work & $4 \times 4$ & Spatial diversity and multiplexing \\
\hline
\end{tabular}

\section{Conclusion}

In this paper, a simple $4 \times 4$ beamforming network with spatial diversity and multiplexing reconfiguration has been designed for MIMO applications. The network is analyzed by the matrix decomposition technique. For achieving diversity and multiplexing reconfiguration of the proposed network, reconfigurable devices have been designed and applied to the network. As a result, the proposed network can achieve spatial diversity (State 1) from/to spatial and diversity multiplexing (State 2 ) response. As an example, the proposed network has been designed, manufactured, and measured at a working frequency of $4.9 \mathrm{GHz}$. Measured and simulated results are in good agreement. For testing the diversity and multiplexing reconfiguration of the radiation beams, a $1 \times 4$ Yagi antenna array has been designed to connect with the reconfigurable network. Good results indicate the proposed network can be widely used in the 
wireless communication networks for MIMO applications. In the future, the beamforming network will integrate the battery onto the substrate for more convenient applications.

\section{Data Availability}

No data were used to support this study.

\section{Conflicts of Interest}

The authors declare that they have no conflicts of interest.

\section{Acknowledgments}

This work was supported in part by the National Natural Science Foundation of China under Grant 61901255, 61771395, and in part by the Fundamental Research Program of TC2020JC09, 2018JM6085, and in part by the Basic Scientific Research of Central University D5000210622.

\section{References}

[1] Y. Zhang and Y. Li, "A dimension-reduction multibeam antenna scheme with dual integrated butler matrix networks for low-complex massive MIMO systems," IEEE Antennas and Wireless Propagation Letters, vol. 19, no. 11, pp. 1938-1942, 2020.

[2] W. Hong, Z. H. Jiang, and C. Yu, "Multi-beam antenna technologies for 5G wireless communications," IEEE Transactions on Antennas and Propagation, vol. 65, no. 12, pp. 6231-6249, Dec. 2017.

[3] Z. Zhao, J. Wang, W. Hou, Y. Li, and B. Ai, "Optimized scheme of antenna diversity for radio wave coverage in tunnel environment," IEEE Access, vol. 8, Article ID 127233, 2020.

[4] Y. Cao, K. S. Chin, W. Che, W. Yang, and E. S. Li, "A compact $38 \mathrm{GHz}$ multibeam antenna array with multifolded butler matrix for 5G applications," IEEE Antennas and Wireless Propagation Letters, vol. 16, pp. 2996-2999, 2017.

[5] L. Zheng and D. N. C. Tse, "Diversity and multiplexing: a fundamental tradeoff in multiple-antenna channels," IEEE Transactions on Information Theory, vol. 49, no. 5, pp. 1073-1096, 2003.

[6] H. Zhai, L. Xi, Y. Zang, and L. Li, “A low profile dual-polarized high isolation MIMO antenna arrays for wideband base station applications," IEEE Transactions on Antennas and Propagation, vol. 66, no. 1, pp. 191-202, 2018.

[7] X. Ding, Z. Zhao, Y. Yang, Z. Nie, and Q. H. Liu, "Wideband quad-polarization reconfigurable antenna using switchable feed network with stable unidirectional radiation patterns," IEEE Access, vol. 6, Article ID 73443, 2018.

[8] V. Palazzi, F. Alimenti, P. Mezzanotte, and L. Roselli, "Novel magnitude and phase reconfigurable $1 \times 4$ RF power distribution network," IEEE Transactions on Microwave Theory and Techniques, vol. 69, no. 1, pp. 29-42, 2021.

[9] M. Zhu and C.-T. M. Wu, "Reconfigurable non-foster elements and squint-free beamforming networks using active transversal filter-based negative group delay circuit," IEEE Transactions on Microwave Theory and Techniques, vol. 70, 2022.

[10] K. Hossain, T. Sabapathy, M. Jusoh et al., "Pattern-Reconfigurable PCB-Based Phased Array for WLAN Applications," in Proceedings of the 2020 nineth Asia-Pacific Conference on
Antennas and Propagation (APCAP), pp. 1-2, Xiamen, China, August 2020.

[11] H. Zhou, J. Geng, J. Li et al., "Novel Beam Scanning Antenna System Fed by Reconfigurable Beamforming Network," in Proceedings of the 2018 International Applied Computational Electromagnetics Society Symposium - China (ACES), pp. 1-2, Beijing, China, August 2018.

[12] L. Sun, G. Zhang, and B. Sun, "Method of synthesizing orthogonal beam-forming networks using QR decomposition," IEEE Access, vol. 7, pp. 325-331, 2019.

[13] J. Yao, C. Lee, and S. P. Yeo, "Microstrip branch-line couplers for crossover application," IEEE Transactions on Microwave Theory and Techniques, vol. 59, no. 1, pp. 87-92, 2011.

[14] https://www.macom.com/products/product-detail/MEST2G010-20. 